Proceedings of the Edinburgh Mathematical Society (2003) 46, 159-168 (C)

DOI:10.1017/S001309150200038X Printed in the United Kingdom

\title{
MULTIPLE SOLUTIONS FOR A CLASS OF QUASILINEAR ELLIPTIC PROBLEMS
}

\author{
JOÃO MARCOS DO Ó ${ }^{1}$ AND PEDRO UBILLA ${ }^{2}$ \\ ${ }^{1}$ Departamento de Matemática, Universidade Fededral da Paraíba, \\ 58059-900 João Pessoa-PB, Brazil (jmbo@mat.ufpb.br) \\ ${ }^{2}$ Universidad de Santiago de Chile, Casilla 30\%, \\ Correo 2, Santiago, Chile (pubilla@lauca.usach.cl)
}

(Received 25 January 2002)

Abstract We deal with a class of $p$-Laplacian Dirichlet boundary-value problems where the combined effects of 'sublinear' and 'superlinear' growths allow us to establish the existence of at least two positive solutions.

Keywords: p-Laplacian; positive solutions; fixed-point index; superlinear problems

AMS 2000 Mathematics subject classification: Primary 35J25; 35J60

Secondary 35J65; 35J70

\section{Introduction}

The objective of this paper is to establish the existence of two radial solutions for the quasilinear boundary-value problem

$$
\left.\begin{array}{rlrl}
-\Delta_{p} u & =f(u) & & \text { in } \Omega, \\
u>0 & & \text { in } \Omega, \\
u & =0 & & \text { on } \partial \Omega,
\end{array}\right\}
$$

where $\Omega \subset \mathbb{R}^{N}$ is a ball of radius $b$, and where $\Delta_{p} u=\operatorname{div}\left(|\nabla u|^{p-2} \nabla u\right)$ is the $p$-Laplacian with $1<p<N$. We will assume that the function $f:[0,+\infty) \rightarrow[0,+\infty)$ is a given continuous function satisfying $f(0)=0$ and the following two conditions:

$\left(\mathrm{H}_{1}\right) \lim _{t \rightarrow 0} f(t) / t^{p-1}=+\infty$, and

$\left(\mathrm{H}_{2}\right) \lim _{t \rightarrow+\infty} f(t) / t^{p-1}=+\infty$.

It follows from assumptions $\left(\mathrm{H}_{1}\right)$ and $\left(\mathrm{H}_{2}\right)$ that there exists $R>0$ such that

$$
\frac{f(R)}{R^{p-1}}=\min _{t>0} \frac{f(t)}{t^{p-1}}
$$


Let $\bar{R}$ be a point where $f$ attains its maximum on the interval $(0, R]$. We will assume the following two further conditions:

$\left(\mathrm{H}_{3}\right) f(\bar{R}) / \bar{R}^{p-1}<\eta=(p /(p-1))^{p-1} N / b^{p}$, and

$\left(\mathrm{H}_{4}\right)$ there exist increasing functions $g_{1}, g_{2} \in C([0,+\infty),[0,+\infty))$ and positive constants $\delta, \eta$, with $\delta \in(0,1)$, such that for all $t>0$,

$$
\begin{aligned}
& g_{2}(t) \leqslant \eta g_{1}(\delta t), \\
& g_{1}(t) \leqslant f(t) \leqslant g_{2}(t) .
\end{aligned}
$$

Our main result is Theorem 1.1, which will be proved in $\S 3$ using fixed-point techniques.

Theorem 1.1. Under the assumptions $\left(\mathrm{H}_{1}\right)-\left(\mathrm{H}_{4}\right)$, problem (1.1) has at least two radial solutions.

Our study was motivated by some recent work on elliptic problems with concaveconvex nonlinearities (see $[\mathbf{1}-\mathbf{3}, \mathbf{9}, \mathbf{1 1}, \mathbf{1 2}]$ ). Ambrosetti et al. $[\mathbf{1}]$ study the second-order elliptic problem

$$
\left.\begin{array}{rlrl}
-\Delta u & =\lambda u^{s}+u^{r} & & \text { in } \Omega, \\
u>0 & & \text { in } \Omega, \\
u & =0 & & \text { on } \partial \Omega,
\end{array}\right\}
$$

where $\Omega$ is a bounded domain in $\mathbb{R}^{N}$ (for $N \geqslant 3$ ) with smooth boundary $\partial \Omega, \Delta$ is the Laplace operator, $\lambda$ is a positive real parameter, and $0<s<1<r$. They prove that there exists a positive real constant $\Lambda$ such that, for all $0<\lambda<\Lambda$, the problem (1.2) has a solution, which is found using subsolution as well as supersolution methods. Here the essential term is $u^{s}$, while the exponent $r$ may be arbitrary. Using variational methods, a second solution of (1.2) is found. In this case, the term $u^{r}$ plays a fundamental role, where $r$ must satisfy $r \leqslant(N+2) /(N-2)$. Among others, the following question is left open. Suppose that $r>(N+2) /(N-2)$ and that $\Omega$ is a ball. Does the problem (1.2) have two positive solutions for $\lambda$ small enough? In [12], it is proved that the assertion is true.

Difficulties arise while extending the study of the problem (1.2) to the $p$-Laplacian operator. Many known techniques and results for the Laplacian no longer apply for the $p$-Laplacian due to its nonlinear nature. Using a radial setting, a priori estimates and topological arguments, Ambrosetti et al. [2] obtained a global multiplicity result for elliptic problems of the form

$$
\left.\begin{array}{rlrl}
-\Delta_{p} u & =\lambda u^{s}+u^{r} & & \text { in } \Omega, \\
u>0 & & \text { in } \Omega, \\
u & =0 & & \text { on } \partial \Omega .
\end{array}\right\}
$$

More precisely, they prove that there is $\Lambda>0$ such that there exist at least two positive solutions of the problem (1.3) in the interval $(0, \Lambda)$, where $\Omega$ is a ball and $0<s<$ $p-1<r<p^{*}=N p /(N-p)$, with $p<N$. In [3], the critical case considering the 
following restrictive assumptions on $p$ is studied: $2 N /(N+2)<p<3$ or $p \geqslant 3$ and $p-1>s>\left(p^{*}+1\right)-2 /(p-1)$. Related results may be found in $[\mathbf{4}, \mathbf{8}]$. For global multiplicity results on a general bounded domain in the subcritical case, see [9]. When $1 \leqslant s<p-1<r \leqslant p^{*}-1$, which includes the critical case, see [11].

Observe that we improve those results for the $p$-Laplacian operator which involve concave and convex nonlinearities because we impose restrictions neither on $p \in(1, N)$ nor on the growth of the nonlinearities, which may be subcritical, critical or supercritical. Note that the nonlinearities we consider are sublinear at 0 and superlinear at $+\infty$, and hence they contain the concave and convex nonlinearities above. We point out that our result is an improvement even in the case studied in [12] because we consider more general nonlinearities. For instance, let $g_{1}(t)=a_{1} t^{s}+b_{1} t^{r} \leqslant g_{2}(t)=a_{2} t^{s}+b_{2} t^{r}$, where $0<s<p-1<r$, and where $a_{1}, b_{1}, a_{2}$ and $b_{2}$ are positive constants. Assume that $g_{1}(t)=a_{1} t^{s}+b_{1} t^{r} \leqslant f(t) \leqslant g_{2}(t)=a_{2} t^{s}+b_{2} t^{r}$. It is easy to see that $f$ satisfies the hypotheses of Theorem 1.1. Finally, note that, in [6], De Figueiredo and Lions studied the Laplacian operator with subcritical nonlinearities that satisfy a sublinearity condition at 0 and a superlinearity condition at $+\infty$.

The paper is organized as follows: $\S 2$ contains preliminary results and $\S 3$ is devoted to proving our main result, Theorem 1.1.

\section{Preliminary results}

We will establish radial solutions of the problem (1.1). In fact, we will obtain solutions $u=u(r)$ of the ordinary equation

$$
\left.\begin{array}{rlrl}
-\left(r^{N-1} \phi\left(u^{\prime}\right)\right)^{\prime} & =r^{N-1} f(u) & & \text { in }(0, b), \\
u & >0 & & \text { in }(0, b), \\
u(b) & =u^{\prime}(0)=0, &
\end{array}\right\}
$$

where $\phi(t)=|t|^{p-2} t$. Performing the change of variable $t=a(r)$, define $z(t)=u(r(t))$, where $a:[0, b) \rightarrow[0,+\infty)$ is given by

$$
a(r)=\frac{p-1}{N-p}\left[r^{(p-N) /(p-1)}-b^{(p-N) /(N-1)}\right] .
$$

Thus (2.1) can be rewritten as

$$
\left.\begin{array}{rlrl}
-\left(\phi\left(z^{\prime}(t)\right)\right)^{\prime} & =r^{(N-1) p /(p-1)}(t) f(z(t)) & & \text { in }(0,+\infty), \\
z & >0 & & \text { in }(0,+\infty), \\
z(0) & =z^{\prime}(+\infty)=0 . & &
\end{array}\right\}
$$

Integrating (2.2) and using the boundary conditions we obtain

$$
\phi\left(z^{\prime}(t)\right)=\int_{t}^{+\infty} r^{(N-1) p /(p-1)}(\tau) f(z(\tau)) \mathrm{d} \tau,
$$


which is equivalent to

$$
z^{\prime}(t)=\left[\int_{t}^{+\infty} r^{(N-1) p /(p-1)}(\tau) f(z(\tau)) \mathrm{d} \tau\right]^{1 /(p-1)} .
$$

Integrating once again we obtain

$$
z(t)=\int_{0}^{t}\left[\int_{s}^{+\infty} G(\tau) f(z(\tau)) \mathrm{d} \tau\right]^{1 /(p-1)} \mathrm{d} s,
$$

where

$$
G(\tau)=\left(b^{(p-N) /(p-1)}+\tau \frac{N-p}{p-1}\right)^{p(1-N) /(N-p)} .
$$

Consequently, we will solve (2.1) using fixed-point techniques. For this, we state the following well-known abstract result without proof (cf. $[\mathbf{5}, \mathbf{7}, \mathbf{1 0}])$.

Lemma 2.1. Let $X$ be a Banach space with norm $|\cdot|$, and let $K \subset X$ be a cone in $X$. For $r>0$, define $K_{r}=K \cap B[0, r]$, where $B[0, r]=\{x \in X:|x| \leqslant r\}$ is the closed ball of radius $r$ centred at the origin of $X$. Assume that $F: K_{r} \rightarrow K$ is a compact map such that $F x \neq x$, for all $x \in \partial K_{r}=\{x \in K:|x|=r\}$.

Then the following assertions hold.

(1) If $|x| \leqslant|F x|$ for all $x \in \partial K_{r}$, then $\imath\left(F, K_{r}, K\right)=0$.

(2) If $|x| \geqslant|F x|$ for all $x \in \partial K_{r}$, then $\imath\left(F, K_{r}, K\right)=1$.

Now we consider the space

$$
X=\{z:[0,+\infty) \rightarrow \mathbb{R}: z \text { is a bounded, continuous function }\}
$$

endowed with the norm $|z|_{\infty}=\sup \{|z(t)|: t \in[0,+\infty)\}$. Let $A: K_{1} \rightarrow X$ be the operator defined by

$$
(A z)(t)=\int_{0}^{t}\left[\int_{s}^{+\infty} G(\tau) f(z(\tau)) \mathrm{d} \tau\right]^{1 /(p-1)} \mathrm{d} s,
$$

where $K_{1}$ is the cone defined by

$$
K_{1}=\{z \in X: z \text { is non-negative, concave and } z(0)=0\} .
$$

Note that the elements of $K_{1}$ are increasing functions.

Lemma 2.2. $A$ is well defined, $A\left(K_{1}\right) \subset K_{1}$, and $A$ is a completely continuous operator.

Proof. For all $s \geqslant 0$, note that

$$
\int_{s}^{+\infty} G(\tau) \mathrm{d} \tau=\frac{1}{N} G(s)^{N(p-1) / p(N-1)}
$$


and that

$$
\int_{0}^{+\infty}\left[\int_{s}^{+\infty} G(\tau) \mathrm{d} \tau\right]^{1 /(p-1)} \mathrm{d} s=\eta^{1 /(1-p)} .
$$

Hence $A$ is well defined.

Also, note that the function $(A z)(t)$ is of class $C^{2}$, whose derivatives are given by

$$
\begin{aligned}
\frac{\mathrm{d}}{\mathrm{d} t}(A z)(t) & =\left[\int_{t}^{+\infty} G(\tau) f(z(\tau)) \mathrm{d} \tau\right]^{1 /(p-1)}, \\
\frac{\mathrm{d}^{2}}{\mathrm{~d} t^{2}}(A z)(t) & =\frac{1}{1-p} G(t)\left[\frac{\mathrm{d}}{\mathrm{d} t}(A z)(t)\right]^{p-2} f(z(t)) .
\end{aligned}
$$

Thus $(A z)(t)$ is increasing and concave. Therefore, $A\left(K_{1}\right) \subset K_{1}$.

It remains to prove that $A$ is a completely continuous operator. Let $\left|z_{n}\right|_{\infty} \leqslant C_{0}$, and let $M_{1}=\max \left\{f(t): t \in\left[0, C_{0}\right]\right\}$. It follows that

$$
\begin{aligned}
\left|\left(A z_{n}\right)(t)\right| & \leqslant M_{1}^{1 /(p-1)} \int_{0}^{+\infty}\left[\int_{s}^{+\infty} G(\tau) \mathrm{d} \tau\right]^{1 /(p-1)} \mathrm{d} s \\
\left|\frac{\mathrm{d}}{\mathrm{d} t}\left(A z_{n}\right)(t)\right| & \leqslant\left[M_{1} \int_{0}^{+\infty} G(\tau) \mathrm{d} \tau\right]^{1 /(p-1)} .
\end{aligned}
$$

By the Arzelá-Ascoli compactness criterion for uniform convergence, there exists a uniformly convergent subsequence $\left(A z_{n_{k}}\right) \subset\left(A z_{n}\right)$ on compact subsets of $[0,+\infty)$. To prove that there exists a uniformly convergent subsequence of $\left(A z_{n}\right)$ it suffices to recall that given $\epsilon>0$, there is $T=T(\epsilon)$ such that

$$
\int_{T}^{+\infty}\left[\int_{s}^{+\infty} G(\tau) \mathrm{d} \tau\right]^{1 /(p-1)} \mathrm{d} s<\epsilon .
$$

We now verify that $A$ is continuous. Let $\left(z_{n}\right) \in X$ such that $\left|z_{n}-z_{0}\right|_{\infty} \rightarrow 0$ as $n \rightarrow \infty$. Thus

$$
\left|\left(A z_{n}\right)(t)-\left(A z_{0}\right)(t)\right| \leqslant \int_{0}^{+\infty}\left|\Gamma_{n}(s)-\Gamma_{0}(s)\right| \mathrm{d} s,
$$

where

$$
\Gamma_{n}(s)=\int_{s}^{+\infty} G(\tau) f\left(z_{n}(\tau)\right) \mathrm{d} \tau \quad \text { and } \quad \Gamma_{0}(s)=\int_{s}^{+\infty} G(\tau) f\left(z_{0}(\tau)\right) \mathrm{d} \tau .
$$

It follows from $\left|z_{n}-z_{0}\right|_{\infty} \rightarrow 0$ that $\Gamma_{n}(s) \rightarrow \Gamma_{0}(s)$ and that

$$
\Gamma_{n}(s) \leqslant \frac{C}{N G(s)^{N(p-1) / p(N-1)}}
$$

for all $s \in[0,+\infty)$. By the Lebesgue-dominated convergence theorem,

$$
\left|A z_{n}-A z_{0}\right|_{\infty} \rightarrow 0
$$

which implies that $A$ is continuous. 
Given $\omega \in K_{1}$, there clearly exists a unique $\tau=\tau(\omega)$ such that $2 \omega(\tau)=|\omega|_{\infty}$.

Define

$$
\tau^{*}=\sup \left\{\tau(A(z)): z \in K_{1}\right\}
$$

and

$$
K=\left\{z \in K_{1}: 2 \inf _{t \geqslant \tau^{*}} z(t) \geqslant|z|_{\infty}\right\}
$$

Lemma 2.3. $\tau^{*}$ is a positive real number and $K$ is an invariant cone by $A$.

The proof is based on the following assertion.

Assertion 2.4. $\left\{\omega /|\omega|_{\infty}: \omega \in A\left(K_{1}\right) \backslash\{0\}\right\}$ is a relatively compact subset of $X$.

Proof. Since $\left\{A z /|A z|_{\infty}: z \in K_{1}\right.$ and $\left.A z \neq 0\right\}$ is a bounded subset of $X$, it suffices to prove that

$$
\left\{[A z]^{\prime} /|A z|_{\infty}: z \in K_{1} \text { and } A z \neq 0\right\}
$$

is also a bounded subset of $X$.

Integrating by parts we have

$$
\begin{aligned}
{\left[\frac{[A z]^{\prime}(t)}{|A z|_{\infty}}\right]^{p-1} } & =\frac{\int_{t}^{+\infty} G(\tau) f(z(\tau) \mathrm{d} \tau}{\left[\int_{0}^{+\infty}\left[\int_{s}^{+\infty} G(\tau) f(z(\tau)) \mathrm{d} \tau\right]^{1 /(p-1)} \mathrm{d} s\right]^{p-1}} \\
& =\frac{(p-1)^{p-1} \int_{t}^{+\infty} G(\tau) f(z(\tau)) \mathrm{d} \tau}{\left[\int_{0}^{+\infty}\left[\int_{s}^{+\infty} G(\tau) f(z(\tau)) \mathrm{d} \tau\right]^{(2-p) /(p-1)} s G(s) f(z(s)) \mathrm{d} s\right]^{p-1}}
\end{aligned}
$$

We consider two cases.

Case $1(1<\boldsymbol{p}<\mathbf{2})$. In this case, it follows from condition $\left(\mathrm{H}_{4}\right)$ that

$$
\begin{aligned}
{\left[\frac{[A z]^{\prime}(t)}{|A z|_{\infty}}\right]^{p-1} } & \leqslant \frac{(p-1)^{p-1} \int_{0}^{+\infty} G(\tau) g_{2}(z(\tau)) \mathrm{d} \tau}{\left[\int_{0}^{+\infty}\left[\int_{s}^{+\infty} G(\tau) g_{1}(z(\tau)) \mathrm{d} \tau\right]^{(2-p) /(p-1)} s G(s) g_{1}(z(s)) \mathrm{d} s\right]^{p-1}} \\
& \leqslant \frac{(p-1)^{p-1} \int_{0}^{+\infty} G(\tau) g_{2}(z(\tau)) \mathrm{d} \tau}{\left[\int_{0}^{+\infty}\left[\int_{s}^{+\infty} G(\tau) \mathrm{d} \tau\right]^{(2-p) /(p-1)} s G(s) g_{1}(z(s))^{1 /(p-1)} \mathrm{d} s\right]^{p-1}} \\
& \leqslant I_{1}+I_{2},
\end{aligned}
$$

where $I_{1}$ and $I_{2}$ are given by

$$
I_{1}=\frac{(p-1)^{p-1} \int_{0}^{1} G(\tau) g_{2}(z(\tau)) \mathrm{d} \tau}{\left[\int_{0}^{1}\left[\int_{s}^{+\infty} G(\tau) \mathrm{d} \tau\right]^{(2-p) /(p-1)} s G(s) g_{1}(z(s))^{1 /(p-1)} \mathrm{d} s\right]^{p-1}}
$$

and

$$
I_{2}=\frac{(p-1)^{p-1} \int_{1}^{+\infty} G(s) g_{2}(z(s)) \mathrm{d} s}{\left[\int_{1}^{+\infty}\left[\int_{s}^{+\infty} G(\tau) \mathrm{d} \tau\right]^{(2-p) /(p-1)} s G(s) g_{1}(z(s))^{1 /(p-1)} \mathrm{d} s\right]^{p-1}}
$$

We estimate each integral separately. 
To estimate $I_{1}$, we use condition $\left(\mathrm{H}_{4}\right)$ to obtain

$$
\begin{aligned}
I_{1} & =\frac{(p-1)^{p-1} \int_{0}^{1} G(\tau) g_{2}(z(\tau)) \mathrm{d} \tau}{\left[\int_{0}^{1}\left[\int_{s}^{+\infty} G(\tau) \mathrm{d} \tau\right]^{(2-p) /(p-1)} s G(s) g_{1}(z(s))^{1 /(p-1)} \mathrm{d} s\right]^{p-1}} \\
& \leqslant \frac{(p-1)^{p-1} \int_{0}^{1} G(\tau) g_{2}(z(\tau)) \mathrm{d} \tau}{\left[\int_{\delta}^{1}\left[\int_{s}^{+\infty} G(\tau) \mathrm{d} \tau\right]^{(2-p) /(p-1)} s G(s) g_{1}(z(\delta))^{1 /(p-1)} \mathrm{d} s\right]^{p-1}} \\
& \leqslant \frac{(p-1)^{p-1} \int_{0}^{1} G(\tau) g_{2}(z(1)) \mathrm{d} \tau}{\left[\int_{\delta}^{1}\left[\int_{s}^{+\infty} G(\tau) \mathrm{d} \tau\right]^{(2-p) /(p-1)} s G(s) g_{1}(\delta z(1))^{1 /(p-1)} \mathrm{d} s\right]^{p-1}} \\
& \leqslant \frac{\eta(p-1)^{p-1} \int_{0}^{1} G(\tau) \mathrm{d} \tau}{\left[\int_{\delta}^{1}\left[\int_{s}^{+\infty} G(\tau) \mathrm{d} \tau\right]^{(2-p) /(p-1)} s G(s) \mathrm{d} s\right]^{p-1}} .
\end{aligned}
$$

To estimate $I_{2}$, we use again condition $\left(\mathrm{H}_{4}\right)$ to get

$$
\begin{aligned}
{\left[\int_{s}^{+\infty} G(\tau) \mathrm{d} \tau\right]^{(2-p) /(p-1)} s G(s) } & \left(g_{1}(z(s))\right)^{1 /(p-1)} \\
& \geqslant N^{(p-2) /(p-1)}\left[s G(s) g_{1}(z(s))\right]^{1 /(p-1)} \\
& \geqslant N^{(p-2) /(p-1)}\left[s G(s) g_{1}(\delta z(s))\right]^{1 /(p-1)} \\
& \geqslant \frac{N^{(p-2) /(p-1)}}{\eta^{1 /(p-1)}}\left[s G(s) g_{2}(z(s))\right]^{1 /(p-1)}
\end{aligned}
$$

which implies

$$
\begin{aligned}
I_{2} & =\frac{(p-1)^{p-1} \int_{1}^{+\infty} G(s) g_{2}(z(s)) \mathrm{d} s}{\left[\int_{1}^{+\infty}\left[\int_{s}^{+\infty} G(\tau) \mathrm{d} \tau\right]^{(2-p) /(p-1)} s G(s) g_{1}(z(s))^{1 /(p-1)} \mathrm{d} s\right]^{p-1}} \\
& \leqslant \frac{\eta(p-1)^{p-1} \int_{1}^{+\infty} G(s) g_{2}(z(s)) \mathrm{d} s}{N^{p-2}\left[\int_{1}^{+\infty}\left[s G(s) g_{2}(z(s))\right]^{1 /(p-1)} \mathrm{d} s\right]^{p-1}} \\
& =\frac{\eta(p-1)^{p-1} \int_{1}^{+\infty} s(1 / s) G(s) g_{2}(z(s)) \mathrm{d} s}{N^{p-2}\left[\int_{1}^{+\infty}\left[s G(s) g_{2}(z(s))\right]^{1 /(p-1)} \mathrm{d} s\right]^{p-1}} \\
& \leqslant \frac{\eta(p-1)^{p-1}\|1 / s\|_{L^{1 /(2-p)}[1,+\infty)}}{N^{p-2}}
\end{aligned}
$$

Case $2(\boldsymbol{p} \geqslant \mathbf{2})$. In this case, in accordance with conditions $(2.6)$ and $\left(\mathrm{H}_{4}\right)$ :

$$
\begin{aligned}
\frac{[A z]^{\prime}(t)}{|A z|_{\infty}} & \leqslant \frac{(p-1) \int_{0}^{+\infty} G(s) f(z(s)) \mathrm{d} s}{\int_{0}^{+\infty} G(s) s f(z(s)) \mathrm{d} s} \\
& \leqslant(p-1)\left[\frac{\int_{0}^{1} G(s) f(z(s)) \mathrm{d} s}{\int_{0}^{1} G(s) s f(z(s)) \mathrm{d} s}+1\right]
\end{aligned}
$$




$$
\begin{aligned}
& \leqslant(p-1)\left[\frac{\int_{0}^{1} G(s) g_{2}(z(s)) \mathrm{d} s}{\int_{0}^{1} G(s) s g_{1}(z(s)) \mathrm{d} s}+1\right] \\
& \leqslant(p-1)\left[\frac{\int_{0}^{1} G(s) g_{2}\left(z\left(s_{M}\right)\right) \mathrm{d} s}{\int_{\delta}^{1} s G(s) g_{1}\left(z\left(s_{m}\right)\right) \mathrm{d} s}+1\right],
\end{aligned}
$$

where $z\left(s_{M}\right)=\max \{z(s): s \in[0,1]\}$ and $z\left(s_{m}\right)=\min \{z(s): s \in[\delta, 1]\}$. It now follows from the fact that $z\left(s_{m}\right) \geqslant \delta z\left(s_{M}\right)$ and condition $\left(\mathrm{H}_{4}\right)$ that

$$
\frac{[A z]^{\prime}(t)}{|A z|_{\infty}} \leqslant(p-1)\left[\eta \frac{\int_{0}^{1} G(s) \mathrm{d} s}{\int_{\delta}^{1} s G(s) \mathrm{d} s}+1\right] .
$$

The result follows by the Arzelá-Ascoli compactness criterion.

Proof of Lemma 2.3. We first show that $\tau^{*}$ is a positive real number. Suppose on the contrary that $\tau^{*}=+\infty$. Then there must exist a sequence $\left(z_{n}\right) \subset K_{1} \backslash\{0\}$ such that $\left(\tau\left(z_{n} /\left|z_{n}\right|_{\infty}\right)\right)$ is a strictly increasing sequence of positive real numbers converging to $+\infty$. By Assertion 2.4, there exists a subsequence of $\left(z_{n} /\left|z_{n}\right|_{\infty}\right)$, which is denoted by the same index, such that $\left(z_{n} /\left|z_{n}\right|_{\infty}\right)$ converges to some $\omega_{0}$ in $X$. Hence $\left|\omega_{0}\right|_{\infty}=1$ and, for large $n$, we must have

$$
\tau\left(z_{n} /\left|z_{n}\right|\right)>\tau\left(\omega_{0}\right)
$$

Note that $\omega_{0}(t) \leqslant \frac{1}{2}$, for all $t \in\left[0, \tau\left(\omega_{0}\right)\right]$. On the other hand, given $t>\tau\left(\omega_{0}\right)$, we have $t<\tau\left(z_{n} /\left|z_{n}\right|\right)$ for large $n$. It follows that

$$
\omega_{0}(t)=\lim _{n \rightarrow+\infty} z_{n}(t) /\left|z_{n}\right|_{\infty} \leqslant \frac{1}{2}, \quad \text { for } t>\tau\left(\omega_{0}\right)
$$

We conclude that $\omega_{0}(t) \leqslant \frac{1}{2}$, for all $t \geqslant 0$. But this is impossible, since $\left|\omega_{0}\right|_{\infty}=1$.

That $K$ is an invariant cone by $A$ is clear. The proof of the lemma is now complete.

Lemma 2.5. We have $\imath\left(A, K_{R}, K\right)=1$.

Proof. According to condition $\left(\mathrm{H}_{3}\right)$, for $z \in \partial K_{R}$,

$$
\begin{aligned}
|A z|_{\infty} & =\max _{t \geqslant 0} \int_{0}^{t}\left[\int_{s}^{+\infty} G(\tau) f(z(\tau)) \mathrm{d} \tau\right]^{1 /(p-1)} \mathrm{d} s \\
& \leqslant \int_{0}^{+\infty}\left[\int_{s}^{+\infty} G(\tau) f(\bar{R}) \mathrm{d} \tau\right]^{1 /(p-1)} \mathrm{d} s \\
& =\frac{f(\bar{R})^{1 /(p-1)}(p-1) b^{p /(p-1)}}{p N^{1 /(p-1)}} \\
& <\bar{R} .
\end{aligned}
$$

Since $\bar{R} \leqslant R$, we have $|A z|_{\infty}<R=|z|_{\infty}$. The result now follows from Lemma 2.1 (2).

Lemma 2.6. There is $r_{1} \in(0, R)$ such that $\imath\left(A, K_{r_{1}}, K\right)=0$. 
Proof. According to condition $\left(\mathrm{H}_{1}\right)$, given $M>0$ there exists $r_{1} \in(0, R)$ such that

$$
f(t) \geqslant M t^{p-1}, \quad \text { for all } t \in\left[0, r_{1}\right] .
$$

Thus for $z \in \partial K_{r_{1}}$,

$$
\begin{aligned}
(A z)\left(\tau^{*}\right) & =\int_{0}^{\tau^{*}}\left[\int_{s}^{+\infty} G(\tau) f(z(\tau)) \mathrm{d} \tau\right]^{1 /(p-1)} \mathrm{d} s \\
& \geqslant \int_{0}^{\tau^{*}}\left[\int_{s}^{+\infty} G(\tau) M z(\tau)^{p-1} \mathrm{~d} \tau\right]^{1 /(p-1)} \mathrm{d} s \\
& \geqslant \int_{0}^{\tau^{*}}\left[\int_{\tau^{*}}^{+\infty} G(\tau) M z(\tau)^{p-1} \mathrm{~d} \tau\right]^{1 /(p-1)} \mathrm{d} s \\
& \geqslant\left[\int_{\tau^{*}}^{+\infty} G(\tau) \mathrm{d} \tau\right]^{1 /(p-1)} \frac{\tau^{*} M^{1 /(p-1)}}{2}|z|_{\infty} .
\end{aligned}
$$

Choosing $M>0$ such that

$$
\tau^{*} G\left(\tau^{*}\right)^{N / p(N-1)}\left[\frac{M}{N}\right]^{1 /(p-1)}>2,
$$

we have that $|A z|_{\infty}>|z|_{\infty}$, for all $z \in \partial K_{r_{1}}$. The result now follows from Lemma 2.1 (1).

Lemma 2.7. There is $r_{2}>R$ such that $\imath\left(A, K_{r_{2}}, K\right)=0$.

Proof. It follows from condition $\left(\mathrm{H}_{2}\right)$ that there exists $r_{3}>R$ such that

$$
f(t) \geqslant M t^{p-1}, \quad \text { for all } t \geqslant r_{3} .
$$

Note that for $z \in \partial K_{2 r_{3}}$ we have

$$
2 \min _{t \geqslant \tau^{*}} z(t) \geqslant|z|_{\infty}=2 r_{3}
$$

which implies

$$
f(z(t)) \geqslant M z(t)^{p-1}, \quad \text { for all } t \geqslant \tau^{*} .
$$

Thus

$$
\begin{aligned}
(A z)\left(\tau^{*}\right) & =\int_{0}^{\tau^{*}}\left[\int_{s}^{+\infty} G(\tau) f(z(\tau)) \mathrm{d} \tau\right]^{1 /(p-1)} \mathrm{d} s \\
& \geqslant \int_{0}^{\tau^{*}}\left[\int_{\tau^{*}}^{+\infty} G(\tau) f(z(\tau)) \mathrm{d} \tau\right]^{1 /(p-1)} \mathrm{d} s \\
& \geqslant \int_{0}^{\tau^{*}}\left[\int_{\tau^{*}}^{+\infty} G(\tau) M z(\tau)^{p-1} \mathrm{~d} \tau\right]^{1 /(p-1)} \mathrm{d} s \\
& \geqslant \tau^{*} G\left(\tau^{*}\right)^{N / p(N-1)}\left[\frac{M}{N}\right]^{1 /(p-1)} \frac{|z|_{\infty}}{2} .
\end{aligned}
$$

Define the number $r_{2}=2 r_{3}$. By (2.7), we have $|A z|_{\infty}>|z|_{\infty}$, for $z \in \partial K_{r_{2}}$, and the result now follows from Lemma $2.1(1)$. 


\section{Proof of the main result}

Proof of Theorem 1.1. It follows from Lemmas 2.5-2.7 and the additivity of the fixed-point index that

$$
\imath\left(A, K_{R} \backslash K_{r_{1}}, K_{r_{1}}\right)=1
$$

and that

$$
\imath\left(A, K_{r_{2}} \backslash K_{R}, K_{R}\right)=-1 .
$$

Consequently, the operator $A$ has two fixed points, namely $z_{1}$ in $K_{R} \backslash K_{r_{1}}$ and $z_{2}$ in $K_{r_{2}} \backslash K_{R}$.

Acknowledgements. J.M.d.Ó. has been partly supported by CNPq, PRONEXMCT/Brazil and the Millennium Institute for the Global Advancement of Brazilian Mathematics (IM-AGIMB). Part of this work was supported by DICYT-USACH and by FONDECYT grant no. 1990183. P.U. thanks the Departamento de Matemática of UFPB for its warm hospitality during his visit in December 1999. The authors also thank the Departamento de Matemática of IMECC-UNICAMP for its warm hospitality during their visit in July 2001.

\section{References}

1. A. Ambrosetti, H. Brezis and C. Cerami, Combined effects of concave and convex nonlinearities in some problems, J. Funct. Analysis 122 (1994), 519-543.

2. A. Ambrosetti, J. Garcia and I. Peral, Multiplicity of solutions for semilinear and quasilinear elliptic problems, J. Funct. Analysis 137 (1996), 219-242.

3. A. Ambrosetti, J. Garcia And I. Peral, Quasilinear equations with a multiple bifurcation, Diff. Integ. Eqns 24 (1997), 37-50.

4. L. Boccardo, M. Escobeto And I. Peral, A Dirichlet problem involving critical exponents, Nonlin. Analysis 24 (1995), 1639-1648.

5. D. G. DE Figueiredo, Positive solutions of semilinear elliptic equations, Lecture Notes in Mathematics, no. 957, pp. 34-87 (Springer, 1982).

6. D. G. DE Figueiredo AND P.-L. Lions, On pairs of positive solutions for a class of semilinear elliptic problems, Indiana Univ. Math. J. 34 (1985), 591-606.

7. K. Deimling, Nonlinear functional analysis (Springer, 1985).

8. J. Garcia AND I. Peral, Some results about the existence of a second positive solution in a quasilinear critical problem, Indiana Univ. Math. J. 43 (1994), 941-957.

9. J. Garcia, J. MAnfredi And I. Peral, Sobolev versus Hölder minimizers and global multiplicity for some quasilinear elliptic equations, Commun. Contemp. Math. 2 (2000), 385-404.

10. D. Guo And V. LAKShmikantham, Nonlinear problem in abstract cones (Academic, 1988).

11. Y. X. Huang, Positive solutions of certain elliptic equations involving critical Sobolev exponents, Nonlin. Analysis 33 (1988), 617-636.

12. R. MA, On a conjecture concerning the multiplicity of positive solutions of elliptic problems, Nonlin. Analysis 27 (1996), 775-780. 